\title{
Sex- and age-specific percentiles of body composition indices for Chinese adults using dual-energy $X$-ray absorptiometry
}

\author{
Zeyu Xiao $^{1} \cdot$ Bin Guo $^{1} \cdot$ Jian Gong $^{1} \cdot$ Yongjin Tang $^{1} \cdot$ Jingjie Shang $^{1} \cdot$ Yong Cheng $^{1} \cdot$ \\ Hao Xu $\mathbf{X}^{1}$
}

Received: 22 October 2015 / Accepted: 21 July 2016 / Published online: 29 July 2016

(C) The Author(s) 2016. This article is published with open access at Springerlink.com

\begin{abstract}
Purpose The aims of the study were to develop sex- and age-specific percentiles for lean mass index (LMI), appendicular LMI (aLMI), fat mass index (FMI), and body fat distribution indices in Chinese adults using dual-energy $\mathrm{X}$-ray absorptiometry (DXA), and to compare those indices with those of other ethnicities using the US NHANES data. Methods Whole-body and regional lean mass and fat mass (FM) were measured using DXA in 5688 healthy males $(n=1693)$ and females $(n=3995)$ aged 20-90 years. Body fat distribution indices were expressed as $\%$ fat trunk $/ \%$ fat legs, trunk/appendicular FM ratio (FMR), and android/gynoid FMR. Percentile curves of LMI, aLMI, FMI, and body fat distribution indices were obtained by the Lambda-Mu-Sigma method.

Results The aLMI and LMI were negatively associated with age, decreasing from the fifth decade for males, but were not associated with age in females. Females had more total FM than males, whereas males had greater central adiposity ( $\%$ fat trunk $/ \%$ fat legs ratio, trunk/appendicular FMR, and android/gynoid FMR) than females. Moreover, FMI and body fat distribution indices consistently increased with age in both sexes, especially in women. In comparison with white, black, and Mexican populations in the USA, Chinese adults had lower total FM, but had greater central adiposity ( $\%$ fat trunk $/ \%$ fat legs ratio and trunk/appendicular FMR). Additionally, older white and Mexican populations showed greater decreases for aLMI and LMI than their Chinese counterparts.
\end{abstract}

Hao Xu

txh@jnu.edu.cn

1 Department of Nuclear Medicine, the First Affiliated Hospital, Jinan University, Guangzhou, China
Conclusions We present the sex- and age-specific percentiles for aLMI, LMI, FMI, and body fat distribution indices by DXA in Chinese adults, which may refine the individual assessment of the nutritional status of Chinese adults.

Keywords Body composition - Percentile curves . Body fat distribution $\cdot$ Nutritional assessment . Dual-energy X-ray absorptiometry
Abbreviations
DXA Dual-energy X-ray absorptiometry
LM Lean mass
FM Fat mass
LMI Lean mass index
FMI Fat mass index
aLMI Appendicular lean mass index
FMR Fat mass ratio
A/G Android/gynoid
$\%$ BF Percentage of body fat mass

\section{Introduction}

Assessment of nutritional status provides a useful predictor of health risk and an opportunity to monitor the effects of nutrition-related disease progression and nutritional intervention in public health and clinical nutrition $[1,2]$. BMI has been widely used in epidemiological studies and clinical practice to provide a quick assessment of nutritional status [3]. However, BMI represents the sum of total body composition. The failure to differentiate between lean mass (LM) and fat mass (FM) limits the usefulness of BMI, which may lead to significant misclassification of nutrition status when applied to individuals [4]. To overcome the limitations of BMI and to obtain more phenotypic details, 
body composition analysis (including LM and FM) is necessary. Measurements of LM and FM in absolute values fail to allow appropriate comparisons among subjects of different sizes. Therefore, some studies have proposed the use of lean mass index (LMI) and fat mass index (FMI) normalized to height as superior measures of nutritional status [5]. In previous studies, FMI was widely used to screen individuals with obesity-risk diseases [6,7], and LMI (especially in limbs) has been applied as an important index for diagnosis of sarcopenia $[8,9]$.

Dual-energy X-ray absorptiometry (DXA) is an accurate and reliable method for assessing body composition, and is capable of separating body mass into LM and FM [10]. Moreover, DXA has the ability to measure on both a regional and whole-body basis with the advantages of low cost, low radiation dose, and high precision [10]. Recently, reference data based on DXA were published for LMI and FMI (total body and regional) in different ethnic populations [11-13]. The most advanced and comprehensive reference values for body composition are age-, sex-, and ethnicity-specific and have been published as percentiles derived from the US NHANES data [11, 12]. We previously described reference data of body composition in healthy Chinese children and adolescents aged 5-19 years using DXA [14]. To the best of our knowledge, there are currently no DXA-based sex- and age-specific data for LMI and FMI in a population of Chinese adults.

Previous studies have shown that Asian populations experience a higher risk of metabolic and cardiovascular diseases at lower levels of BMI than other ethnic populations $[15,16]$. This paradoxical finding conveyed the necessity for additional adiposity measures to supplement BMI in assessing health risk, such as measures of body fat distribution that are more strongly associated with risk factors for obesity-related diseases than total fat mass [17]. Moreover, ethnic differences in body fat distribution exist in adults $[11,12,18]$. Thus, ethnicity-specific percentile curves of body fat distribution indices would further assist in the assessment of nutritional status in adults.

Therefore, the primary aim of the current study was to develop sex- and age-specific percentiles for LMI, FMI, and body fat distribution indices in a population of Chinese adults aged 20-90 years using DXA. A secondary aim was to compare the body composition indices of the Chinese population with those of other ethnic populations.

\section{Methods}

\section{Study population}

The participants were recruited from a body composition and osteoporosis study at the First Affiliated Hospital of Jinan University (Guangzhou, China) from 2004 to 2012. The present study included healthy Chinese men and women from 20 to 90 years of age who had a BMI of $16-30 \mathrm{~kg} / \mathrm{m}^{2}$. Subjects were included in the study if they were functionally independent Chinese individuals over 20 years of age, who were in apparent good health. Subjects were excluded if they met any of the following criteria: (1) a history of fracture; (2) medication known to affect the musculoskeletal system (e.g., anti-osteoporotic drugs, androgens or anti-androgen drugs, corticosteroids); (3) chronic disease known to affect bone metabolism (e.g., hyperthyroidism, hyperparathyroidism, rheumatoid arthritis, chronic renal insufficiency); (4) metal implants (e.g., pacemakers, joint replacement device); or (5) inability to determine the menstruation state or experiencing nonnatural menopause (natural menopause was designated if there was a complete natural cessation of menses for more than 12 months). Ultimately, 1693 men and 3995 women were included in our study. All subjects provided written informed consent to participate in the study, which was approved by the Ethics Committee of the First Affiliated Hospital, Jinan University.

\section{Anthropometry and body composition measurement}

A research physician obtained information on medical history and medication use in a personal interview. Height and body weight were obtained based on standard methods; height was measured without shoes to the nearest $0.1 \mathrm{~cm}$, and weight with only light clothing to the nearest $0.1 \mathrm{~kg}$. BMI was calculated as body weight divided by height squared $\left(\mathrm{kg} / \mathrm{m}^{2}\right)$. Total and regional LM and FM were obtained through whole-body DXA scans (Lunar Prodigy, GE Healthcare, Madison, WI, USA), and data were analyzed using software version 10.0 provided by the manufacturer. Android and gynoid regions were automatically obtained using the software provided by the manufacturer. The appendicular region is defined as including both the left and right arms and legs area. From these measurements, the following derivative values were calculated: FMI (total fat mass/height ${ }^{2}$ ), LMI (total lean mass/height ${ }^{2}$ ), aLMI (appendicular lean mass/height ${ }^{2}$ ), and \% BF (percentage of body fat mass $=$ total body fat mass/weight $\times 100 \%$ ). Indices of body fat distribution were included in the analysis as previously described: $\%$ fat trunk/\% fat legs, trunk/ appendicular FM ratio (FMR), and android/gynoid (A/G) FMR. Daily quality assurance scans were performed by scanning the spine phantom according to the manufacturer's instructions. All DXA measurements were taken by the same trained technologist throughout the study. The precision error (\% CV) was less than $2 \%$ for total LM and total FM and less than $3 \%$ for regional (trunk, appendicular, android, gynoid) LM and FM, as determined by duplicate 
scans with repositioning between each measurement in 30 volunteer subjects.

\section{Statistical analysis}

The values of continuous variables are presented as the mean $\pm \mathrm{SD}$. Unpaired-sample $t$ tests were conducted to evaluate the significance of the mean difference between males and females. Age- and sex-specific percentile curves for aLMI, LMI, FMI, \% BF and indices of body fat distribution were generated using the Lambda-MuSigma method (LMS-chartmaker version Pro 2.3, Medical Research Council, UK). Each percentile (changing distribution) is summarized by three curves representing the skewness (L), the median (M), and the coefficient of variation $(S)$ as these change with the independent variable age [19]. The $Z$-scores can be calculated by the following equation:

$Z=\frac{\mathrm{M}\left(\left(\frac{X}{M}\right)^{L}-1\right)}{L s}$,

where $X$ is the body composition measure of interest.

The 50th percentile curves for aLMI, LMI, FMI, and body fat distribution indices (including $\%$ fat trunk/\% fat legs ratio and trunk/appendicular FMR) of Chinese adults were compared with those of white, black, and Mexican populations in the US NHANES reference data using the same GE-Lunar DXA scanner [12]. The comparisons did not include the A/G FMR because the NHANES reference data did not provide these values. All tests were two-tailed, and a $p$ value of less than 0.05 was considered statistically significant.

\section{Results}

\section{Characteristics of subjects}

Details of the subject characteristics are shown in Table 1. Males and females differed significantly in weight and height as well as in total and regional LM and FM. Males were heavier, were taller, and had higher LM, whereas FM was greater in females.

\section{Percentiles for LMI, FMI, aLMI, and \% BF}

Percentiles (3rd, 10th, 25th, 50th, 75th, 90th, and 97th) for LMI, FMI, aLMI, and \% BF for males and females aged 20-90 years are shown in Tables 2, 3, 4 and 5, and percentile curves are given in Fig. 1. The differences between sexes were seen in percentile curves for LMI, FMI, aLMI, and \% BF. The aLMI and LMI were consistently higher
Table 1 Characteristics of study subjects

\begin{tabular}{|c|c|c|c|}
\hline & $\begin{array}{l}\text { Males } \\
(n=1693) \\
\text { Mean } \pm \text { SD }\end{array}$ & $\begin{array}{l}\text { Females } \\
(n=3995) \\
\text { Mean } \pm \text { SD }\end{array}$ & $\begin{array}{l}\text { Total } \\
(n=5668) \\
\text { Mean } \pm \text { SD }\end{array}$ \\
\hline Age (years) & $52.8 \pm 17.5^{\mathrm{c}}$ & $54.0 \pm 15.9$ & $53.6 \pm 16.4$ \\
\hline Weight (kg) & $63.5 \pm 10.3^{\mathrm{c}}$ & $53.8 \pm 8.1$ & $56.7 \pm 9.9$ \\
\hline Height, m & $1.68 \pm 0.06^{\mathrm{c}}$ & $1.57 \pm 0.05$ & $1.60 \pm 0.08$ \\
\hline BMI $\left(\mathrm{kg} / \mathrm{m}^{2}\right)$ & $22.5 \pm 3.2^{\mathrm{b}}$ & $21.9 \pm 3.0$ & $22.1 \pm 3.1$ \\
\hline \multicolumn{4}{|c|}{ Body composition measures $(\mathrm{kg})$} \\
\hline Total LM & $46.72 \pm 6.00^{c}$ & $33.93 \pm 3.78$ & $37.73 \pm 7.32$ \\
\hline Total FM & $14.27 \pm 6.47^{\mathrm{c}}$ & $17.86 \pm 5.65$ & $16.80 \pm 6.13$ \\
\hline Total BMC & $2.527 \pm 0.433^{\mathrm{c}}$ & $1.978 \pm 0.384$ & $2.142 \pm 0.472$ \\
\hline Trunk LM & $22.39 \pm 2.64^{\mathrm{c}}$ & $16.76 \pm 2.04$ & $18.44 \pm 3.41$ \\
\hline Trunk FM & $8.62 \pm 4.30^{c}$ & $9.76 \pm 3.48$ & $9.42 \pm 3.78$ \\
\hline Appendicular LM & $20.52 \pm 3.11^{\mathrm{c}}$ & $14.12 \pm 1.97$ & $16.03 \pm 3.76$ \\
\hline Appendicular FM & $5.07 \pm 2.19^{\mathrm{b}}$ & $7.39 \pm 2.35$ & $6.70 \pm 2.54$ \\
\hline \multicolumn{4}{|c|}{ Body composition index $\left(\mathrm{kg} / \mathrm{m}^{2}\right)$} \\
\hline Total LMI & $16.6 \pm 1.6^{\mathrm{c}}$ & $13.8 \pm 1.3$ & $14.6 \pm 1.9$ \\
\hline Total FMI & $5.1 \pm 2.2^{\mathrm{a}}$ & $7.3 \pm 2.2$ & $6.6 \pm 2.5$ \\
\hline Appendicular LMI & $7.3 \pm 0.9^{c}$ & $5.7 \pm 0.7$ & $6.2 \pm 1.0$ \\
\hline \multicolumn{4}{|l|}{ Body fat distribution } \\
\hline$\% \mathrm{BF}$ & $21.62 \pm 7.62^{c}$ & $32.55 \pm 6.49$ & $29.30 \pm 8.48$ \\
\hline $\begin{array}{l}\% \text { Fat trunk } / \% \text { fat } \\
\text { legs ratio }\end{array}$ & $1.33 \pm 0.30^{\mathrm{c}}$ & $1.08 \pm 0.19$ & $1.16 \pm 0.25$ \\
\hline $\begin{array}{l}\text { Trunk/appendicu- } \\
\text { lar FMR }\end{array}$ & $1.66 \pm 0.43^{\mathrm{c}}$ & $1.33 \pm 0.34$ & $1.43 \pm 0.39$ \\
\hline A/G FMR & $0.63 \pm 0.19^{c}$ & $0.51 \pm 0.15$ & $0.54 \pm 0.17$ \\
\hline \multicolumn{4}{|l|}{ BMI status (\%) } \\
\hline $\mathrm{BMI}<18.5 \mathrm{~kg} / \mathrm{m}^{2}$ & 12.3 & 13.8 & 13.4 \\
\hline $\mathrm{BMI} \geq 25 \mathrm{~kg} / \mathrm{m}^{2}$ & 23.3 & 16.2 & 18.4 \\
\hline
\end{tabular}

$B M I$ body mass index, $L M$ lean mass, $F M$ fat mass, $B M C$ bone mineral content, $L M I$ lean mass index, $F M I$ fat mass index, \% $B F$ percentage of body fat mass, $F M R$ fat mass ratio, $A / G$ android/gynoid

a $P \geq 0.05 ;{ }^{\text {b }} P<0.01 ;{ }^{\mathrm{c}} P<0.001$. Compared with females (unpaired-sample $t$ tests)

in males of all age groups than females, whereas the FMI and $\% \mathrm{BF}$ were consistently higher in females. The aLMI $(r=-0.123, P<0.001)$ and LMI $(r=-0.292, P<0.001)$ were negatively associated with age and started to decrease from the fifth decade in men, whereas in women the aLMI and LMI had no correlation with age, although a slow decrease from the sixth decade was seen for aLMI. The FMI and \% BF were positively related to age and showed a steady increase with age in both sexes.

Comparisons with the ethnic groups (including whites, blacks, and Mexicans) of US counterparts from NHANES reference data for the 50th percentile curves of LMI, FMI, and aLMI using the GE-Lunar DXA scanner are shown in Fig. 2. Compared with the white, black, and Mexican populations in the USA, the 50th percentiles of LMI, FMI, and aLMI were consistently distinctly lower in Chinese males 


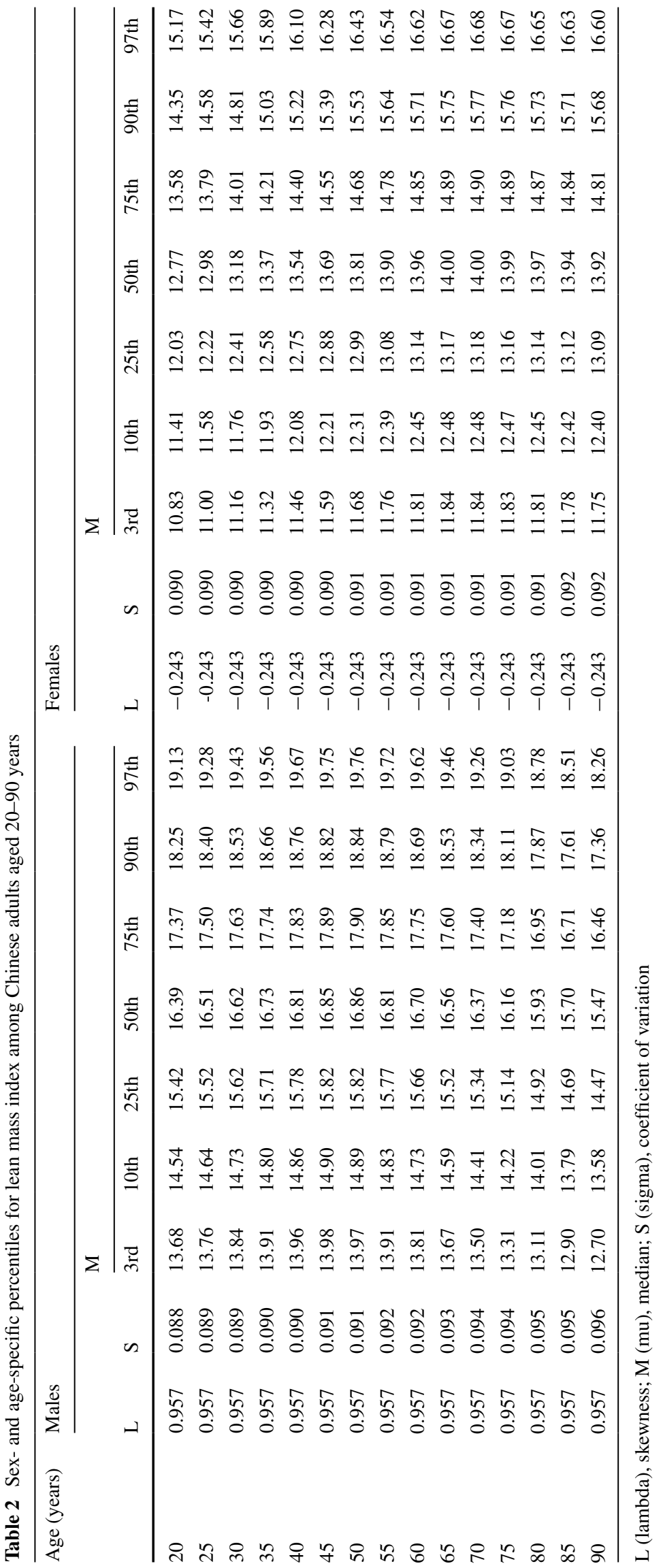


Table 3 Sex- and age-specific percentiles for fat mass index among Chinese adults aged 20-90 years

\begin{tabular}{|c|c|c|c|c|c|c|c|c|c|c|c|c|c|c|c|c|c|c|}
\hline \multirow[t]{3}{*}{ Age (years) } & \multicolumn{9}{|l|}{ Males } & \multicolumn{9}{|c|}{ Females } \\
\hline & \multirow[b]{2}{*}{$\mathrm{L}$} & \multirow[b]{2}{*}{ S } & \multicolumn{6}{|l|}{ M } & \multirow[b]{2}{*}{ 97th } & \multirow[b]{2}{*}{$\mathrm{L}$} & \multirow[b]{2}{*}{$S$} & \multicolumn{7}{|l|}{ M } \\
\hline & & & $3 \mathrm{rd}$ & 10th & 25 th & 50th & 75th & 90th & & & & $3 \mathrm{rd}$ & 10th & 25 th & 50th & 75th & 90th & 97th \\
\hline 20 & 0.748 & 0.553 & 0.50 & 1.37 & 2.43 & 3.76 & 5.22 & 6.63 & 8.11 & 0.641 & 0.300 & 2.85 & 3.70 & 4.63 & 5.75 & 6.96 & 8.11 & 9.31 \\
\hline 25 & 0.748 & 0.537 & 0.60 & 1.51 & 2.61 & 3.98 & 5.48 & 6.93 & 8.44 & 0.641 & 0.301 & 2.96 & 3.84 & 4.81 & 5.98 & 7.23 & 8.43 & 9.68 \\
\hline 30 & 0.748 & 0.522 & 0.71 & 1.66 & 2.79 & 4.20 & 5.74 & 7.22 & 8.76 & 0.641 & 0.301 & 3.07 & 3.98 & 4.99 & 6.20 & 7.51 & 8.76 & 10.05 \\
\hline 35 & 0.748 & 0.507 & 0.83 & 1.81 & 2.97 & 4.41 & 5.98 & 7.49 & 9.06 & 0.641 & 0.301 & 3.18 & 4.13 & 5.18 & 6.44 & 7.79 & 9.09 & 10.44 \\
\hline 40 & 0.748 & 0.492 & 0.96 & 1.97 & 3.15 & 4.62 & 6.21 & 7.74 & 9.33 & 0.641 & 0.302 & 3.30 & 4.28 & 5.37 & 6.67 & 8.08 & 9.43 & 10.83 \\
\hline 45 & 0.748 & 0.477 & 1.09 & 2.12 & 3.33 & 4.80 & 6.41 & 7.95 & 9.54 & 0.641 & 0.302 & 3.41 & 4.43 & 5.55 & 6.91 & 8.37 & 9.76 & 11.21 \\
\hline 50 & 0.748 & 0.462 & 1.22 & 2.27 & 3.48 & 4.96 & 6.56 & 8.09 & 9.68 & 0.641 & 0.303 & 3.51 & 4.56 & 5.73 & 7.13 & 8.63 & 10.07 & 11.57 \\
\hline 55 & 0.748 & 0.447 & 1.35 & 2.41 & 3.61 & 5.09 & 6.68 & 8.19 & 9.77 & 0.641 & 0.303 & 3.60 & 4.68 & 5.87 & 7.31 & 8.86 & 10.34 & 11.89 \\
\hline 60 & 0.748 & 0.432 & 1.48 & 2.54 & 3.74 & 5.19 & 6.76 & 8.26 & 9.80 & 0.641 & 0.304 & 3.67 & 4.77 & 5.99 & 7.46 & 9.05 & 10.56 & 12.14 \\
\hline 65 & 0.748 & 0.418 & 1.62 & 2.67 & 3.85 & 5.29 & 6.83 & 8.29 & 9.81 & 0.641 & 0.304 & 3.72 & 4.84 & 6.08 & 7.58 & 9.19 & 10.73 & 12.33 \\
\hline 70 & 0.748 & 0.403 & 1.75 & 2.79 & 3.96 & 5.36 & 6.87 & 8.31 & 9.79 & 0.641 & 0.305 & 3.75 & 4.89 & 6.15 & 7.66 & 9.29 & 10.85 & 12.48 \\
\hline 75 & 0.748 & 0.389 & 1.88 & 2.91 & 4.06 & 5.44 & 6.91 & 8.31 & 9.75 & 0.641 & 0.305 & 3.77 & 4.92 & 6.19 & 7.72 & 9.37 & 10.95 & 12.60 \\
\hline 80 & 0.748 & 0.375 & 2.02 & 3.04 & 4.16 & 5.51 & 6.94 & 8.30 & 9.70 & 0.641 & 0.306 & 3.79 & 4.95 & 6.23 & 7.78 & 9.44 & 11.03 & 12.69 \\
\hline 85 & 0.748 & 0.361 & 2.17 & 3.16 & 4.26 & 5.58 & 6.97 & 8.29 & 9.65 & 0.641 & 0.307 & 3.81 & 4.97 & 6.27 & 7.82 & 9.50 & 11.11 & 12.78 \\
\hline 90 & 0.748 & 0.347 & 2.31 & 3.29 & 4.37 & 5.65 & 7.00 & 8.28 & 9.60 & 0.641 & 0.307 & 3.82 & 5.00 & 6.30 & 7.87 & 9.56 & 11.18 & 12.86 \\
\hline
\end{tabular}

L (lambda), skewness; M (mu), median; S (sigma), coefficient of variation

Table 4 Sex- and age-specific percentiles for appendicular lean mass index among Chinese adults aged 20-90 years

\begin{tabular}{|c|c|c|c|c|c|c|c|c|c|c|c|c|c|c|c|c|c|c|}
\hline \multirow[t]{3}{*}{ Age (years) } & \multicolumn{9}{|l|}{ Males } & \multicolumn{9}{|l|}{ Females } \\
\hline & \multirow[b]{2}{*}{$\mathrm{L}$} & \multirow[b]{2}{*}{ S } & \multicolumn{7}{|l|}{ M } & \multirow[b]{2}{*}{$\mathrm{L}$} & \multirow[b]{2}{*}{ S } & \multicolumn{7}{|l|}{ M } \\
\hline & & & $3 \mathrm{rd}$ & 10th & 25 th & 50th & 75 th & 90th & 97th & & & $3 \mathrm{rd}$ & 10th & 25 th & 50th & 75th & 90th & 97th \\
\hline 20 & 1.241 & 0.110 & 5.94 & 6.46 & 6.98 & 7.54 & 8.10 & 8.59 & 9.07 & -0.220 & 0.105 & 4.49 & 4.77 & 5.08 & 5.45 & 5.85 & 6.25 & 6.67 \\
\hline 25 & 1.261 & 0.111 & 5.92 & 6.45 & 6.97 & 7.54 & 8.10 & 8.60 & 9.08 & -0.115 & 0.107 & 4.54 & 4.84 & 5.16 & 5.54 & 5.96 & 6.36 & 6.79 \\
\hline 30 & 1.281 & 0.112 & 5.90 & 6.44 & 6.97 & 7.54 & 8.10 & 8.60 & 9.09 & -0.010 & 0.109 & 4.59 & 4.89 & 5.23 & 5.63 & 6.05 & 6.47 & 6.90 \\
\hline 35 & 1.301 & 0.113 & 5.88 & 6.42 & 6.96 & 7.54 & 8.10 & 8.60 & 9.09 & 0.095 & 0.110 & 4.62 & 4.95 & 5.29 & 5.70 & 6.14 & 6.56 & 7.01 \\
\hline 40 & 1.320 & 0.114 & 5.85 & 6.40 & 6.94 & 7.52 & 8.09 & 8.60 & 9.08 & 0.200 & 0.112 & 4.65 & 4.99 & 5.34 & 5.77 & 6.22 & 6.64 & 7.09 \\
\hline 45 & 1.339 & 0.114 & 5.81 & 6.37 & 6.91 & 7.50 & 8.07 & 8.57 & 9.06 & 0.306 & 0.113 & 4.66 & 5.01 & 5.38 & 5.81 & 6.26 & 6.69 & 7.14 \\
\hline 50 & 1.357 & 0.115 & 5.76 & 6.31 & 6.86 & 7.45 & 8.02 & 8.52 & 9.01 & 0.412 & 0.114 & 4.65 & 5.01 & 5.38 & 5.82 & 6.28 & 6.71 & 7.15 \\
\hline 55 & 1.374 & 0.116 & 5.69 & 6.24 & 6.79 & 7.37 & 7.94 & 8.44 & 8.93 & 0.519 & 0.115 & 4.63 & 5.00 & 5.38 & 5.82 & 6.28 & 6.71 & 7.15 \\
\hline 60 & 1.389 & 0.117 & 5.60 & 6.15 & 6.69 & 7.28 & 7.85 & 8.34 & 8.82 & 0.627 & 0.116 & 4.60 & 4.97 & 5.36 & 5.81 & 6.27 & 6.69 & 7.12 \\
\hline 65 & 1.401 & 0.118 & 5.49 & 6.04 & 6.58 & 7.16 & 7.72 & 8.22 & 8.69 & 0.734 & 0.116 & 4.54 & 4.92 & 5.32 & 5.77 & 6.22 & 6.64 & 7.06 \\
\hline 70 & 1.411 & 0.119 & 5.37 & 5.92 & 6.46 & 7.03 & 7.58 & 8.07 & 8.54 & 0.842 & 0.117 & 4.47 & 4.86 & 5.25 & 5.70 & 6.15 & 6.56 & 6.97 \\
\hline 75 & 1.419 & 0.120 & 5.25 & 5.79 & 6.32 & 6.88 & 7.43 & 7.91 & 8.37 & 0.950 & 0.117 & 4.39 & 4.78 & 5.17 & 5.62 & 6.06 & 6.46 & 6.86 \\
\hline 80 & 1.427 & 0.121 & 5.12 & 5.65 & 6.17 & 6.73 & 7.27 & 7.74 & 8.19 & 1.057 & 0.117 & 4.30 & 4.69 & 5.09 & 5.52 & 5.95 & 6.34 & 6.73 \\
\hline 85 & 1.434 & 0.122 & 4.98 & 5.51 & 6.03 & 6.58 & 7.11 & 7.57 & 8.01 & 1.165 & 0.116 & 4.22 & 4.61 & 5.00 & 5.43 & 5.85 & 6.22 & 6.59 \\
\hline 90 & 1.441 & 0.122 & 4.85 & 5.38 & 5.88 & 6.42 & 6.94 & 7.40 & 7.84 & 1.270 & 0.116 & 4.13 & 4.52 & 4.91 & 5.33 & 5.74 & 6.11 & 6.46 \\
\hline
\end{tabular}

L (lambda), skewness; M (mu), median; S (sigma), coefficient of variation

and females, with the exception of similar values in older Mexican and white populations for aLMI and LMI. Moreover, the 50th percentiles of aLMI and LMI showed relatively greater decreases in older white and Mexican individuals than in Chinese individuals.

\section{Percentiles for body fat distribution indices}

Percentiles (3rd, 10th, 25th, 50th, 75th, 90th, and 97th) for body fat distribution indices (including $\%$ fat trunk $/ \%$ fat legs ratio, trunk/appendicular FMR, and A/G FMR) for 


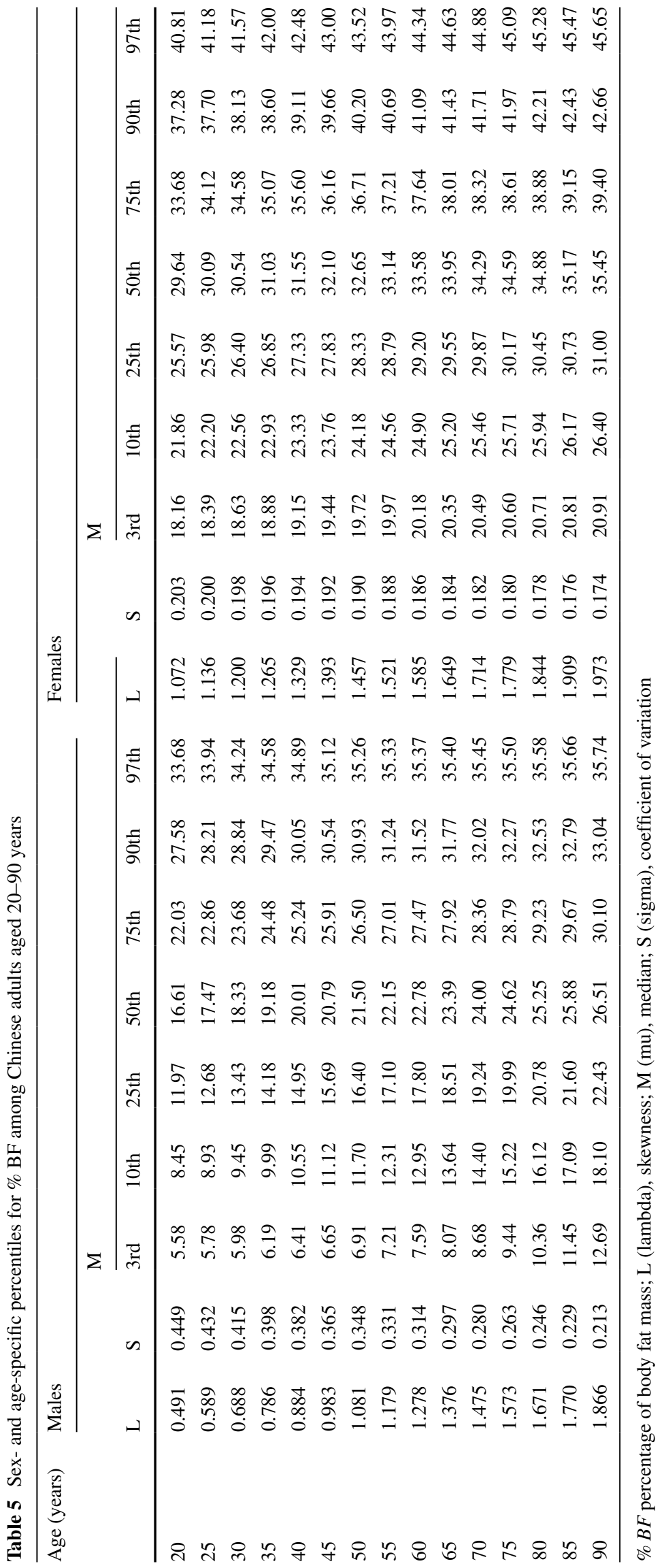


Fig. 1 Percentile curves (the 3rd, 10th, 25th, 50th, 75th, 90th, and 97th) for LMI, FMI, appendicular LMI, and \% body fat in Chinese males and females aged 20-90 years. ( $L M I$ lean mass index, $F M I$ fat mass index, $\%$ $B F$ percentage of body fat mass)
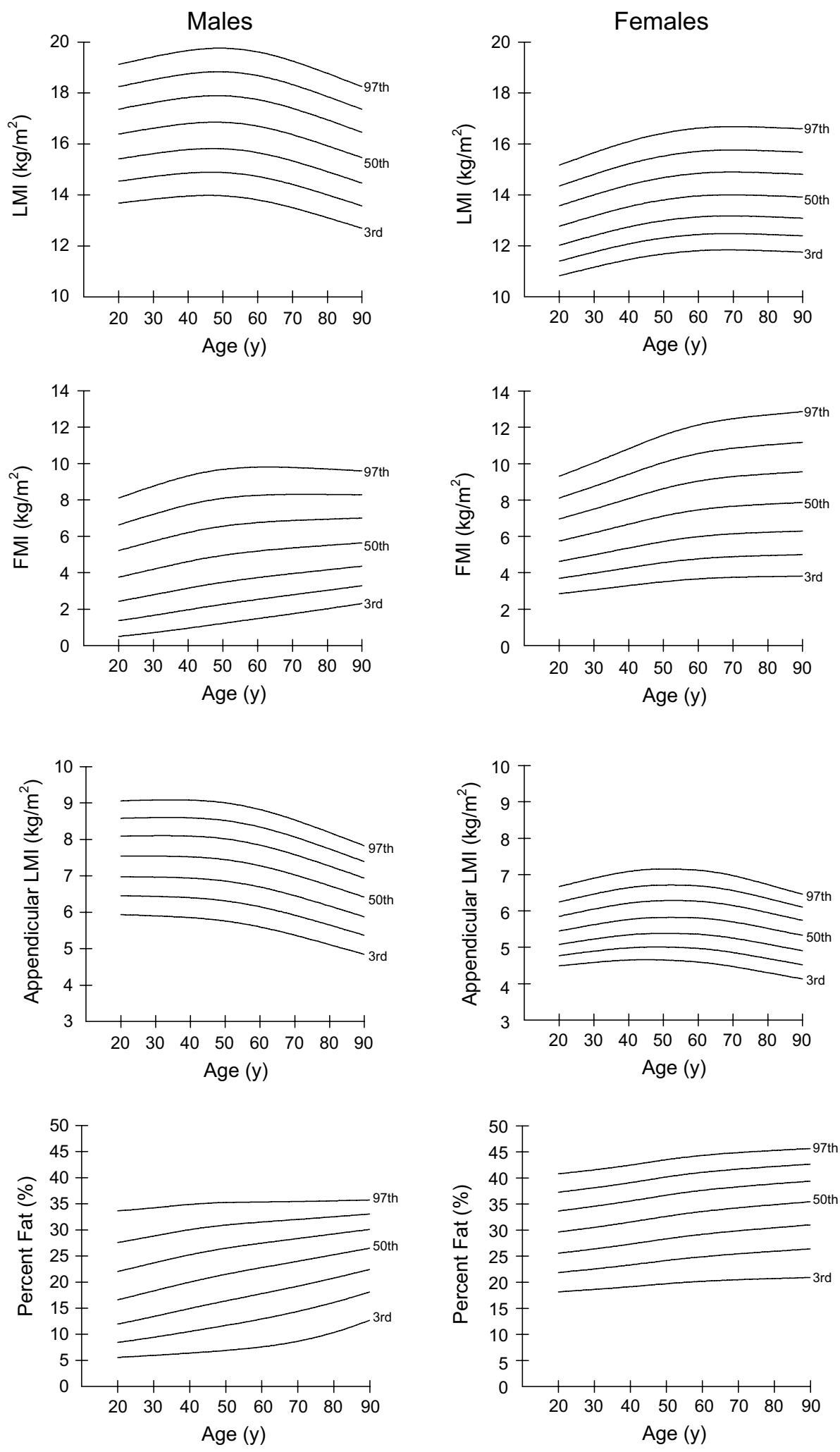

males and females aged 20-90 years are shown in Tables 6, 7 and 8, and percentile curves are given in Fig. 3. The values of $\%$ fat trunk $/ \%$ fat legs ratio, trunk/appendicular FMR, and A/G FMR were consistently higher for males than females. The pattern of changes in the \% fat trunk/\% fat legs ratio, trunk/appendicular FMR, and A/G FMR differed by sex. The $\%$ fat trunk $/ \%$ fat legs ratio, trunk/appendicular FMR, and A/G FMR increased gradually until the 

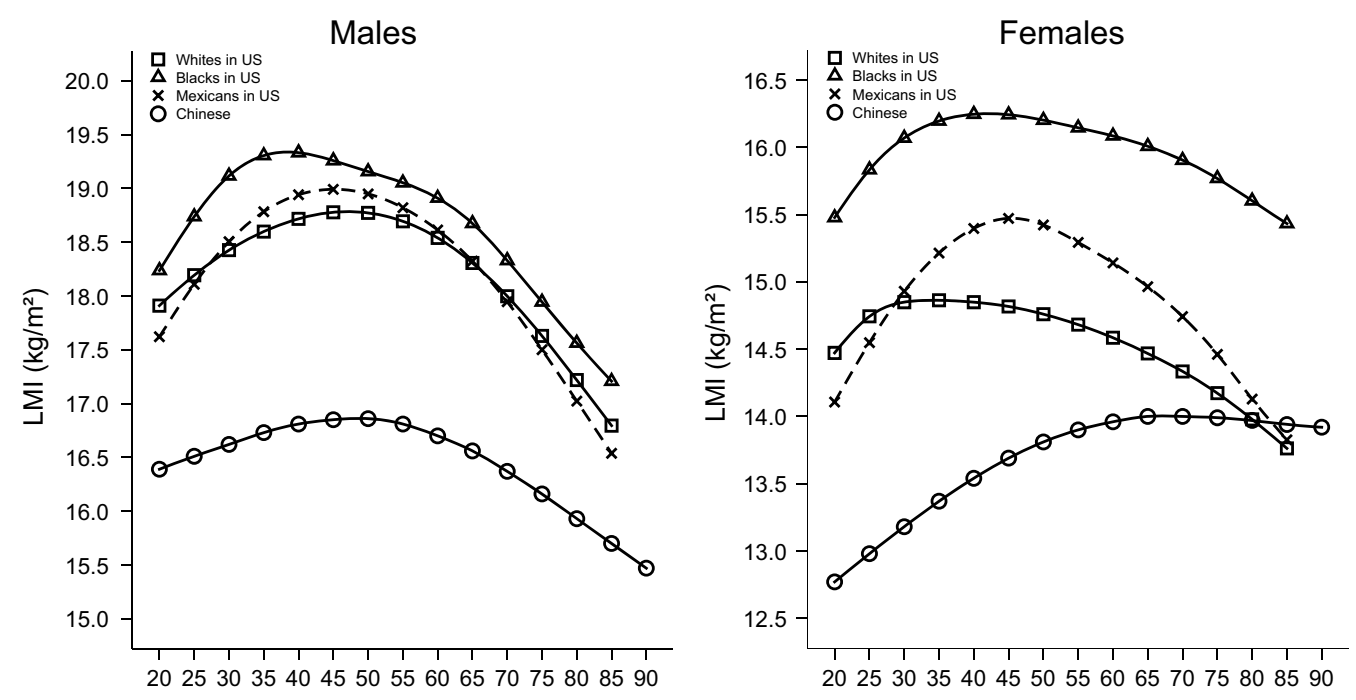

Age (y)
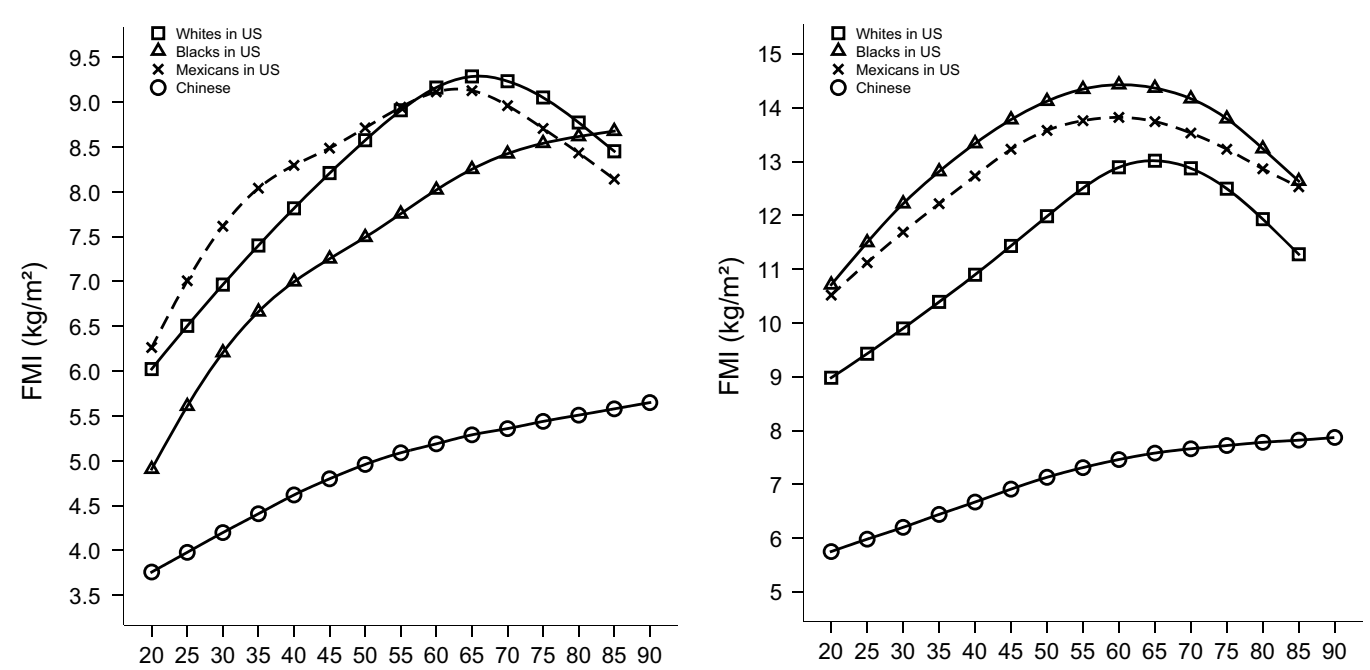

Age (y)

Age (y)
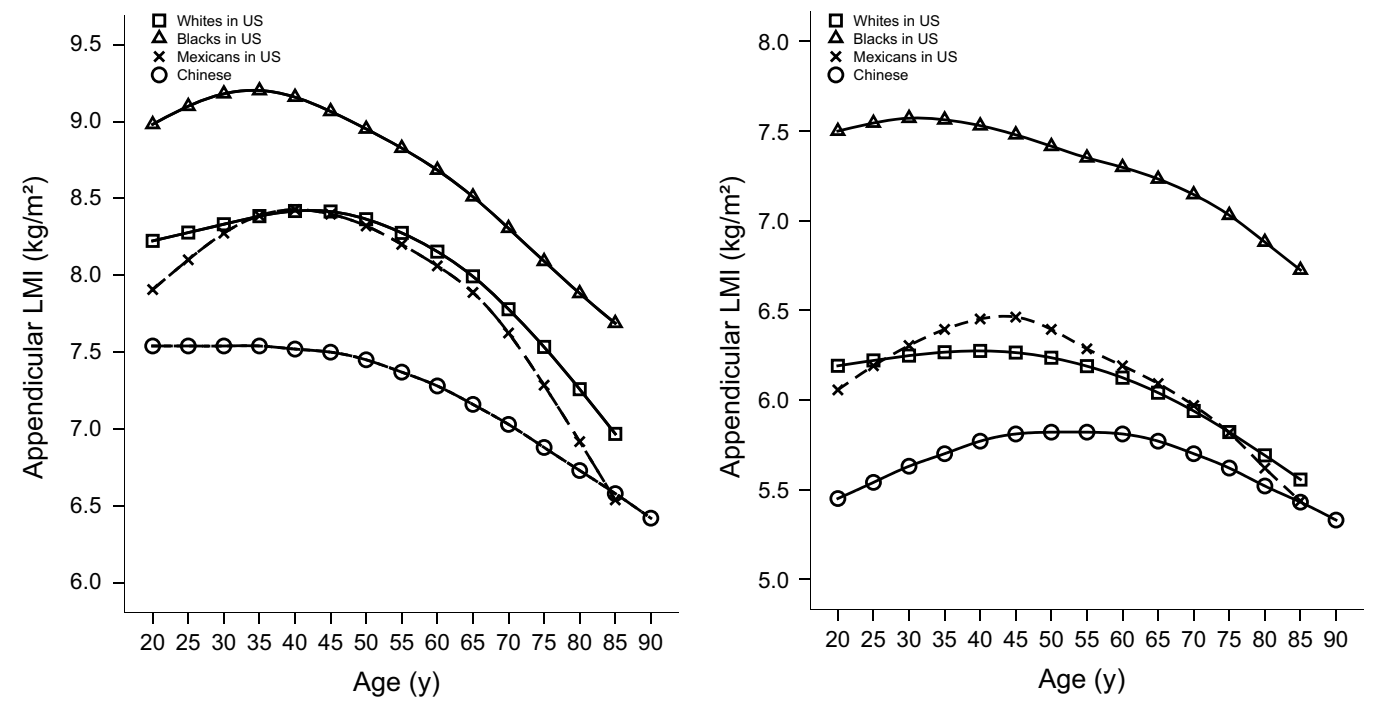

Fig. 2 Comparisons of the 50th percentile curves for LMI, FMI, and appendicular LMI according to age and gender for Chinese versus American adults (including white, black, and Mexican adults) from NHANES data. (LMI lean mass index, FMI fat mass index) 
Table 6 Sex- and age-specific percentiles for \% fat trunk/\% fat legs ratio among Chinese adults aged 20-90 years

\begin{tabular}{|c|c|c|c|c|c|c|c|c|c|c|c|c|c|c|c|c|c|c|}
\hline \multirow[t]{3}{*}{ Age (years) } & \multicolumn{9}{|l|}{ Males } & \multicolumn{9}{|l|}{ Females } \\
\hline & \multirow[b]{2}{*}{$\mathrm{L}$} & \multirow[b]{2}{*}{ S } & \multicolumn{7}{|l|}{ M } & \multirow[b]{2}{*}{$\mathrm{L}$} & \multirow[b]{2}{*}{$S$} & \multicolumn{7}{|l|}{ M } \\
\hline & & & $3 \mathrm{rd}$ & 10th & 25 th & 50th & 75 th & 90th & 97th & & & $3 \mathrm{rd}$ & 10th & 25 th & 50th & 75th & 90th & 97th \\
\hline 20 & 0.989 & 0.200 & 0.68 & 0.82 & 0.95 & 1.10 & 1.25 & 1.38 & 1.51 & 1.341 & 0.136 & 0.70 & 0.79 & 0.87 & 0.96 & 1.05 & 1.12 & 1.20 \\
\hline 25 & 1.094 & 0.204 & 0.71 & 0.85 & 1.00 & 1.16 & 1.32 & 1.46 & 1.60 & 1.232 & 0.140 & 0.71 & 0.80 & 0.88 & 0.97 & 1.06 & 1.14 & 1.22 \\
\hline 30 & 1.195 & 0.208 & 0.72 & 0.89 & 1.05 & 1.23 & 1.40 & 1.55 & 1.69 & 1.120 & 0.143 & 0.72 & 0.80 & 0.89 & 0.99 & 1.08 & 1.17 & 1.25 \\
\hline 35 & 1.286 & 0.211 & 0.74 & 0.93 & 1.10 & 1.29 & 1.47 & 1.63 & 1.78 & 0.999 & 0.147 & 0.73 & 0.82 & 0.91 & 1.01 & 1.11 & 1.19 & 1.28 \\
\hline 40 & 1.355 & 0.212 & 0.76 & 0.96 & 1.15 & 1.34 & 1.53 & 1.69 & 1.85 & 0.857 & 0.150 & 0.74 & 0.83 & 0.92 & 1.03 & 1.13 & 1.23 & 1.32 \\
\hline 45 & 1.397 & 0.212 & 0.77 & 0.98 & 1.18 & 1.38 & 1.57 & 1.74 & 1.90 & 0.684 & 0.154 & 0.76 & 0.85 & 0.94 & 1.05 & 1.16 & 1.27 & 1.37 \\
\hline 50 & 1.410 & 0.211 & 0.79 & 1.00 & 1.20 & 1.41 & 1.60 & 1.77 & 1.93 & 0.481 & 0.158 & 0.78 & 0.87 & 0.97 & 1.08 & 1.20 & 1.31 & 1.42 \\
\hline 55 & 1.397 & 0.209 & 0.80 & 1.01 & 1.21 & 1.42 & 1.61 & 1.78 & 1.94 & 0.272 & 0.162 & 0.80 & 0.89 & 0.99 & 1.10 & 1.23 & 1.35 & 1.48 \\
\hline 60 & 1.362 & 0.207 & 0.81 & 1.02 & 1.21 & 1.41 & 1.61 & 1.77 & 1.93 & 0.093 & 0.167 & 0.82 & 0.90 & 1.00 & 1.12 & 1.25 & 1.38 & 1.53 \\
\hline 65 & 1.321 & 0.205 & 0.82 & 1.01 & 1.20 & 1.40 & 1.59 & 1.76 & 1.91 & -0.023 & 0.171 & 0.82 & 0.90 & 1.00 & 1.12 & 1.26 & 1.40 & 1.55 \\
\hline 70 & 1.290 & 0.204 & 0.81 & 1.00 & 1.18 & 1.37 & 1.56 & 1.72 & 1.87 & -0.074 & 0.176 & 0.81 & 0.89 & 0.99 & 1.12 & 1.26 & 1.40 & 1.56 \\
\hline 75 & 1.276 & 0.202 & 0.79 & 0.97 & 1.15 & 1.34 & 1.51 & 1.67 & 1.82 & -0.076 & 0.180 & 0.79 & 0.88 & 0.98 & 1.10 & 1.24 & 1.39 & 1.55 \\
\hline 80 & 1.276 & 0.202 & 0.77 & 0.94 & 1.11 & 1.29 & 1.47 & 1.62 & 1.76 & -0.047 & 0.185 & 0.76 & 0.85 & 0.95 & 1.08 & 1.22 & 1.37 & 1.53 \\
\hline 85 & 1.280 & 0.203 & 0.74 & 0.91 & 1.08 & 1.25 & 1.42 & 1.56 & 1.71 & -0.004 & 0.190 & 0.74 & 0.83 & 0.93 & 1.06 & 1.20 & 1.35 & 1.51 \\
\hline 90 & 1.285 & 0.203 & 0.71 & 0.88 & 1.04 & 1.21 & 1.37 & 1.51 & 1.65 & 0.043 & 0.195 & 0.71 & 0.80 & 0.91 & 1.03 & 1.18 & 1.32 & 1.49 \\
\hline
\end{tabular}

L (lambda), skewness; M (mu), median; S (sigma), coefficient of variation

Table 7 Sex- and age-specific percentiles for trunk/appendicular fat mass ratio among Chinese adults aged 20-90 years

\begin{tabular}{|c|c|c|c|c|c|c|c|c|c|c|c|c|c|c|c|c|c|c|}
\hline \multirow[t]{3}{*}{ Age (years) } & \multicolumn{9}{|l|}{ Males } & \multicolumn{9}{|l|}{ Females } \\
\hline & \multirow[b]{2}{*}{$\mathrm{L}$} & \multirow[b]{2}{*}{ S } & \multicolumn{7}{|l|}{ M } & \multirow[b]{2}{*}{ L } & \multirow[b]{2}{*}{ S } & \multicolumn{7}{|l|}{ M } \\
\hline & & & $3 \mathrm{rd}$ & 10th & 25 th & 50th & 75th & 90th & 97th & & & $3 \mathrm{rd}$ & 10th & 25 th & 50th & 75th & 90th & 97th \\
\hline 20 & 0.869 & 0.206 & 0.76 & 0.91 & 1.06 & 1.22 & 1.40 & 1.55 & 1.71 & 0.237 & 0.181 & 0.74 & 0.83 & 0.93 & 1.06 & 1.19 & 1.32 & 1.46 \\
\hline 25 & 0.888 & 0.214 & 0.81 & 0.97 & 1.14 & 1.33 & 1.52 & 1.70 & 1.87 & 0.184 & 0.186 & 0.76 & 0.85 & 0.95 & 1.08 & 1.23 & 1.37 & 1.52 \\
\hline 30 & 0.907 & 0.221 & 0.85 & 1.03 & 1.22 & 1.43 & 1.65 & 1.84 & 2.04 & 0.132 & 0.191 & 0.77 & 0.87 & 0.98 & 1.11 & 1.26 & 1.42 & 1.58 \\
\hline 35 & 0.920 & 0.228 & 0.89 & 1.09 & 1.30 & 1.53 & 1.77 & 1.98 & 2.20 & 0.083 & 0.196 & 0.79 & 0.89 & 1.00 & 1.15 & 1.31 & 1.47 & 1.65 \\
\hline 40 & 0.919 & 0.233 & 0.92 & 1.14 & 1.37 & 1.62 & 1.88 & 2.11 & 2.34 & 0.034 & 0.201 & 0.81 & 0.92 & 1.04 & 1.19 & 1.36 & 1.53 & 1.73 \\
\hline 45 & 0.894 & 0.237 & 0.95 & 1.18 & 1.42 & 1.69 & 1.96 & 2.21 & 2.46 & -0.018 & 0.206 & 0.84 & 0.95 & 1.07 & 1.23 & 1.42 & 1.61 & 1.82 \\
\hline 50 & 0.845 & 0.240 & 0.98 & 1.21 & 1.46 & 1.74 & 2.02 & 2.28 & 2.55 & -0.072 & 0.212 & 0.87 & 0.98 & 1.12 & 1.29 & 1.48 & 1.69 & 1.93 \\
\hline 55 & 0.776 & 0.243 & 1.00 & 1.23 & 1.48 & 1.76 & 2.06 & 2.33 & 2.60 & -0.114 & 0.218 & 0.89 & 1.01 & 1.15 & 1.34 & 1.55 & 1.78 & 2.04 \\
\hline 60 & 0.699 & 0.244 & 1.02 & 1.24 & 1.49 & 1.77 & 2.07 & 2.35 & 2.64 & -0.126 & 0.226 & 0.91 & 1.04 & 1.18 & 1.38 & 1.60 & 1.85 & 2.13 \\
\hline 65 & 0.640 & 0.246 & 1.02 & 1.24 & 1.48 & 1.77 & 2.07 & 2.35 & 2.65 & -0.099 & 0.233 & 0.91 & 1.04 & 1.20 & 1.40 & 1.64 & 1.90 & 2.20 \\
\hline 70 & 0.605 & 0.247 & 1.01 & 1.23 & 1.46 & 1.74 & 2.04 & 2.33 & 2.63 & -0.035 & 0.242 & 0.90 & 1.04 & 1.20 & 1.41 & 1.67 & 1.93 & 2.24 \\
\hline 75 & 0.592 & 0.250 & 0.99 & 1.20 & 1.43 & 1.71 & 2.00 & 2.29 & 2.58 & 0.047 & 0.250 & 0.88 & 1.03 & 1.20 & 1.42 & 1.68 & 1.95 & 2.26 \\
\hline 80 & 0.598 & 0.252 & 0.95 & 1.16 & 1.39 & 1.67 & 1.96 & 2.24 & 2.53 & 0.139 & 0.258 & 0.86 & 1.01 & 1.19 & 1.41 & 1.68 & 1.96 & 2.26 \\
\hline 85 & 0.615 & 0.256 & 0.92 & 1.13 & 1.35 & 1.62 & 1.91 & 2.19 & 2.47 & 0.238 & 0.267 & 0.82 & 0.99 & 1.17 & 1.41 & 1.68 & 1.96 & 2.26 \\
\hline 90 & 0.633 & 0.259 & 0.88 & 1.09 & 1.31 & 1.58 & 1.87 & 2.14 & 2.42 & 0.335 & 0.276 & 0.79 & 0.96 & 1.16 & 1.40 & 1.68 & 1.95 & 2.26 \\
\hline
\end{tabular}

L (lambda), skewness; M (mu), median; S (sigma), coefficient of variation

fifth decade in both sexes. These measures decreased thereafter in males, but remained steady in females.

Figure 4 shows the 50th percentile curves of $\%$ BF, \% fat trunk $/ \%$ fat legs ratio and trunk/appendicular FMR using the GE-Lunar DXA scanner for white, black, and
Mexican populations in the USA using NHANES reference data. The 50th percentiles of $\%$ BF were consistently distinctly lower in Chinese males and females; however, body fat distribution indices for Chinese adults were not parallel with US data in terms of total FM for either sex. 
Table 8 Sex- and age-specific percentiles for android/gynoid fat mass ratio among Chinese adults aged 20-90 years

\begin{tabular}{|c|c|c|c|c|c|c|c|c|c|c|c|c|c|c|c|c|c|c|}
\hline \multirow[t]{3}{*}{ Age (years) } & \multicolumn{9}{|l|}{ Males } & \multicolumn{9}{|c|}{ Females } \\
\hline & \multirow[b]{2}{*}{$\mathrm{L}$} & \multirow[b]{2}{*}{ S } & \multicolumn{7}{|l|}{ M } & \multirow[b]{2}{*}{$\mathrm{L}$} & \multirow[b]{2}{*}{$\mathrm{S}$} & \multicolumn{7}{|l|}{ M } \\
\hline & & & $3 \mathrm{rd}$ & 10th & 25 th & 50th & 75th & 90th & 97th & & & 3rd & 10th & 25 th & 50th & 75th & 90th & 97th \\
\hline 20 & 0.095 & 0.274 & 0.24 & 0.28 & 0.34 & 0.41 & 0.49 & 0.58 & 0.67 & 0.544 & 0.230 & 0.22 & 0.26 & 0.31 & 0.36 & 0.42 & 0.48 & 0.54 \\
\hline 25 & 0.235 & 0.274 & 0.26 & 0.31 & 0.37 & 0.45 & 0.54 & 0.63 & 0.73 & 0.529 & 0.232 & 0.23 & 0.27 & 0.32 & 0.38 & 0.44 & 0.50 & 0.56 \\
\hline 30 & 0.375 & 0.273 & 0.28 & 0.34 & 0.41 & 0.49 & 0.59 & 0.69 & 0.79 & 0.516 & 0.234 & 0.24 & 0.28 & 0.33 & 0.39 & 0.46 & 0.52 & 0.58 \\
\hline 35 & 0.513 & 0.271 & 0.30 & 0.37 & 0.45 & 0.54 & 0.64 & 0.74 & 0.85 & 0.506 & 0.237 & 0.25 & 0.29 & 0.34 & 0.41 & 0.47 & 0.54 & 0.61 \\
\hline 40 & 0.645 & 0.270 & 0.32 & 0.39 & 0.48 & 0.58 & 0.69 & 0.80 & 0.91 & 0.501 & 0.239 & 0.26 & 0.31 & 0.36 & 0.43 & 0.50 & 0.57 & 0.64 \\
\hline 45 & 0.763 & 0.268 & 0.33 & 0.42 & 0.51 & 0.62 & 0.74 & 0.84 & 0.95 & 0.493 & 0.241 & 0.27 & 0.32 & 0.38 & 0.45 & 0.53 & 0.60 & 0.68 \\
\hline 50 & 0.866 & 0.267 & 0.34 & 0.43 & 0.54 & 0.65 & 0.77 & 0.88 & 0.99 & 0.478 & 0.245 & 0.28 & 0.34 & 0.40 & 0.48 & 0.56 & 0.64 & 0.72 \\
\hline 55 & 0.948 & 0.265 & 0.34 & 0.45 & 0.55 & 0.67 & 0.79 & 0.90 & 1.01 & 0.462 & 0.249 & 0.30 & 0.36 & 0.43 & 0.51 & 0.60 & 0.68 & 0.77 \\
\hline 60 & 1.005 & 0.264 & 0.35 & 0.46 & 0.57 & 0.69 & 0.81 & 0.92 & 1.03 & 0.453 & 0.253 & 0.31 & 0.38 & 0.45 & 0.54 & 0.63 & 0.72 & 0.82 \\
\hline 65 & 1.040 & 0.264 & 0.35 & 0.46 & 0.58 & 0.70 & 0.83 & 0.94 & 1.05 & 0.453 & 0.257 & 0.32 & 0.39 & 0.47 & 0.56 & 0.66 & 0.76 & 0.86 \\
\hline 70 & 1.061 & 0.264 & 0.35 & 0.47 & 0.58 & 0.71 & 0.83 & 0.95 & 1.05 & 0.467 & 0.262 & 0.33 & 0.40 & 0.48 & 0.58 & 0.68 & 0.79 & 0.90 \\
\hline 75 & 1.076 & 0.265 & 0.35 & 0.46 & 0.58 & 0.71 & 0.84 & 0.95 & 1.06 & 0.493 & 0.268 & 0.33 & 0.40 & 0.49 & 0.59 & 0.70 & 0.81 & 0.92 \\
\hline 80 & 1.093 & 0.268 & 0.34 & 0.46 & 0.58 & 0.71 & 0.84 & 0.95 & 1.06 & 0.527 & 0.273 & 0.33 & 0.41 & 0.49 & 0.60 & 0.71 & 0.83 & 0.94 \\
\hline 85 & 1.114 & 0.272 & 0.33 & 0.46 & 0.58 & 0.71 & 0.84 & 0.95 & 1.06 & 0.565 & 0.279 & 0.33 & 0.41 & 0.50 & 0.61 & 0.73 & 0.84 & 0.96 \\
\hline 90 & 1.137 & 0.275 & 0.32 & 0.45 & 0.57 & 0.71 & 0.84 & 0.95 & 1.06 & 0.603 & 0.285 & 0.32 & 0.41 & 0.50 & 0.62 & 0.74 & 0.86 & 0.98 \\
\hline
\end{tabular}

L (lambda), skewness; M (mu), median; S (sigma), coefficient of variation

Particularly, Chinese men had greater central adiposity (\% fat trunk/\% fat legs ratio and trunk/appendicular FMR). The 50th percentiles of the $\%$ fat trunk $/ \%$ fat legs ratio and trunk/appendicular FMR were consistently higher in Chinese males than their US counterparts, with the exception of similar values in Mexican individuals aged $80-85$ years. The 50th percentiles of $\%$ fat trunk $/ \%$ fat legs ratio were consistently higher in Chinese women than white American women, whereas these measures were lower than those of black and Mexican women, except for black women aged 55-85 years. The 50th percentiles of trunk/appendicular FMR were consistently higher in Chinese women than their American counterparts, with the exception of lower values than Mexican women aged $20-60$ years.

\section{Discussion}

The present study provides sex- and age-specific curves and percentiles for LMI, FMI, aLMI, and body fat distribution indices using DXA in Chinese adults. First, to the best of our knowledge, there are no previous studies establishing values for LMI, FMI, aLMI, and body fat distribution indices using DXA based on a large sample of Chinese adults. Using these percentile values provides more accurate assessments of nutritional status (obesity and sarcopenia) for Chinese men and women. Second, in comparison with white, black, and Mexican populations in the USA as reported in the NHANES reference data [12], Chinese adults had lower total FM than their US counterparts, but regional body fat distribution indices were not parallel with US data in terms of total FM for either sex. Particularly, Chinese men had greater central adiposity (\% fat trunk $/ \%$ fat legs ratio and trunk/appendicular FMR). Moreover, older white and Mexican populations had relatively greater decreases for LMI than those of the surveyed Chinese population.

\section{Values for LMI and FMI by DXA}

In the present study, LM and FM were normalized by height $^{2}$, similar to BMI, which is weight divided by height ${ }^{2}$. LMI, especially aLMI, as a measure of abnormally low muscle mass has been widely applied in the diagnosis of sarcopenia [8, 9]. FMI values can be used to assess clinical obesity as proposed by Kelly [11] for identifying subjects with high obesity disease risks and for enrolling high-risk individuals into clinical trials.

Consistent with previous studies [11-13], our data demonstrate sex differences in LM and FM throughout the entire life span, with males having greater LM and lower FM than females. Moreover, the patterns of age-related changes for LM and FM were different in Chinese men and women. In the present study, the LMI and aLMI for Chinese men were negatively correlated with age and an evident decease was observed from the fifth decade. In Chinese women, LMI and aLMI showed no relationship with 
Fig. 3 Percentile curves (the 3rd, 10th, 25th, 50th, 75th, 90th, and 97 th) for $\%$ fat trunk $/ \%$ fat legs ratio, trunk/appendicular FMR, and A/G FMR in Chinese males and females aged 20-90 years. (FMR fat mass ratio, $A / G$ android/gynoid)
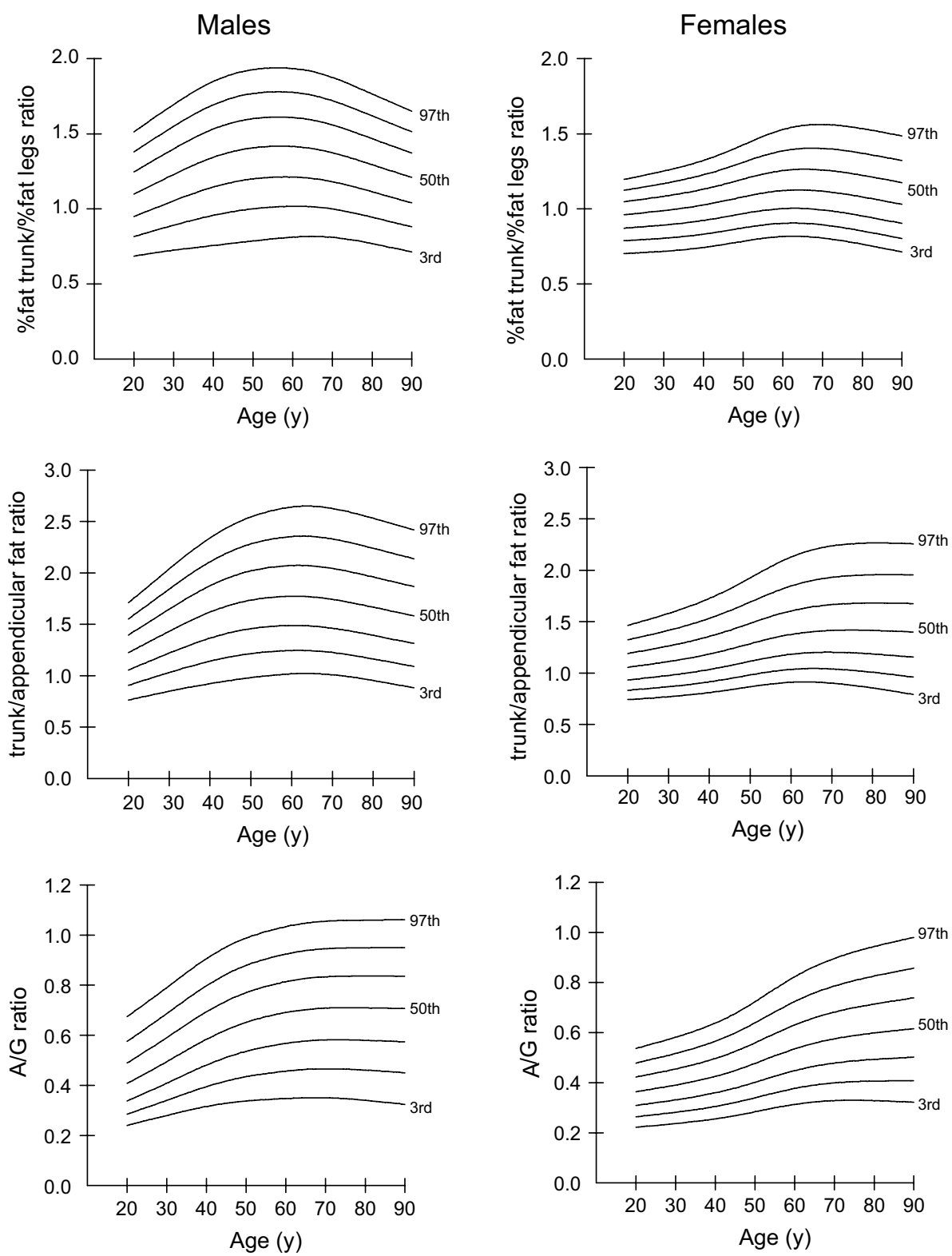

age. Other studies showed both males and females experience age-related deceases in appendicular LM as measured by DXA [20] and whole-body LM as measured by MRI [21], and that males typically experience greater deceases than females for LM. Previous studies in Asian populations have shown the prevalence of sarcopenia in men was higher than that in women [22, 23]; therefore, males may be more likely to develop sarcopenia than females in Asian populations. Consistent with the NHANES data [11, 12], FMI increased with age in Chinese men and women. However, FMI had no relationship with age in Korean men [13]. Sex differences in LM and FM are distinct early in life, and become much more marked during puberty as shown in our previous study [14]. These sex differences in body composition may be mainly attributed to the action of sex steroid hormones, which drive the dimorphisms during pubertal development [24].

Ethnic differences in body composition have been reported in previous studies [11, 12]. Compared with their American counterparts as reported in the NHANES data, the values of LMI, aLMI, and FMI were distinctly lower in Chinese males and females, with the exception of similar values recorded for older Mexican and white individuals for aLMI and LMI. An interesting finding was that in both sexes, older white and Mexican populations had greater deceases for aLMI and LMI than Chinese. Older Chinese adults today may have performed more physical activities in their lifetimes than younger Chinese populations because they may have experienced more difficult living conditions since early adulthood, thus their muscle mass may be in 

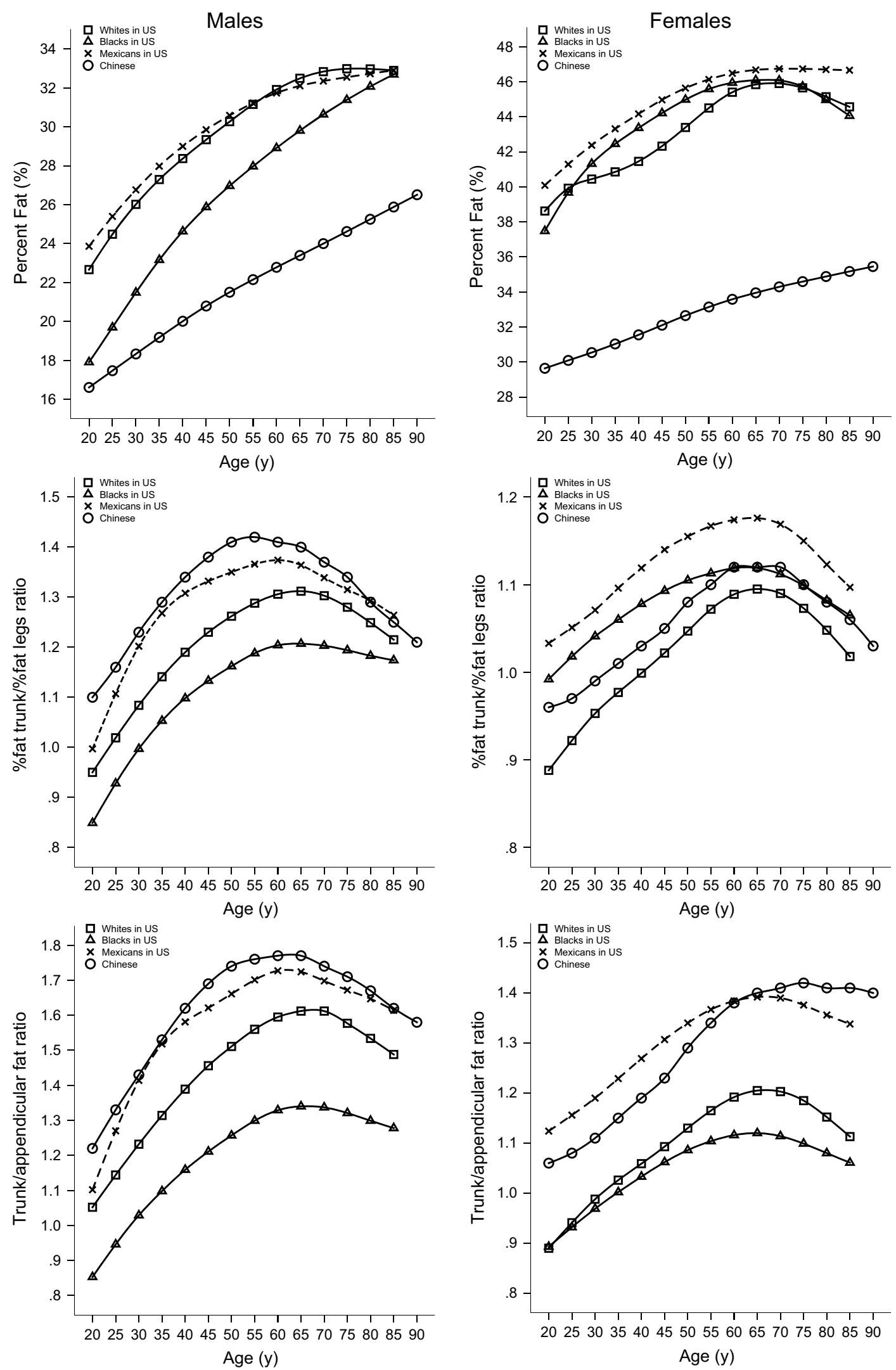

Fig. 4 Comparisons of the 50th percentile curves for \% body fat, \% fat trunk/\% fat legs ratio and trunk/appendicular FMR according to age and gender for Chinese versus American adults (including white, black, and Mexican adults) from NHANES data. (FMR fat mass ratio) 
better condition than that of younger generations. This may have led to the older Chinese having lower deceases for LM than expected.

\section{Values for body fat distribution indices}

Over and above fat mass per se, the pattern of body fat distribution is an independent and stronger predictor of health risk $[17,25]$. Previous studies have revealed that android (namely central, upper body or truncal) adipose deposition is related with an increased risk of metabolic and cardiovascular diseases [17, 26], while gynoid (namely glutealfemoral or lower body) fat tissue is associated with reduced metabolic risk and may be protective against adverse health effects in both sexes [17, 25].

Sex differences in body fat distribution have been well studied. In accordance with previous studies [11-13], we found males accumulated more central adiposity ( $\%$ fat trunk/\% fat legs ratio and trunk/appendicular FMR) than females throughout the entire life span, though females generally have higher total adiposity relative to males. Ethnic differences in fat distribution have been reported previously $[11,12]$, with white and Mexican populations in the USA having relatively higher central fat than black populations. Previous investigations in Asian populations have demonstrated that Asian ethnicity is associated with higher central adiposity than in Caucasian populations [27, 28], even in childhood [29]. Compared with their American counterparts, we discovered Chinese adults had distinctly lower total FM, while the central fat distribution indices were not parallel with the US data in terms of total FM for either sex. Particularly, Chinese men had more central adiposity. The values and patterns of change in the body fat distribution indices in Chinese adults were similar to those of their Korean counterparts [13].

\section{Limitations}

There are several potential limitations in this study. First, the cross-sectional design does not allow for the further longitudinal assessment of body composition accrual in individuals. Second, individuals with extreme underweight $\left(\mathrm{BMI}<16 \mathrm{~kg} / \mathrm{m}^{2}\right)$ or obesity $\left(\mathrm{BMI} \geq 30 \mathrm{~kg} / \mathrm{m}^{2}\right)$ were excluded, and the subjects in this study were not randomly selected, despite the large sample $(n=5688)$. Thus, these data may not be representative of the whole national population. However, we feel that the data were fairly representative of the population in terms of median BMI of both sexes. The median BMI was $22.5 \mathrm{~kg} / \mathrm{m}^{2}$ for males and $22.1 \mathrm{~kg} / \mathrm{m}^{2}$ for females in this study, compared with a BMI of $22.8 \mathrm{~kg} / \mathrm{m}^{2}$ for males and $23.3 \mathrm{~kg} / \mathrm{m}^{2}$ for females in a randomly selected population of the Working Group on Obesity in China [30]. Additionally, estimates of body composition using different DXA scanners may show substantially different DXA values. Our data are limited only to results derived from the Lunar Prodigy DXA; these percentile curves may be improperly used with results from other brands of DXA scanners. Finally, caution should be advised in using the present values because of the differences in nutritional habits, genetic backgrounds, physical activity levels, and other lifestyle factors of the study population.

In conclusion, we present the sex- and age-specific percentiles for aLMI, LMI, FMI, and body fat distribution indices using DXA in Chinese adults. These body composition indices may refine the individual assessment of the nutritional status of adults, and serve as a useful tool for public health screening with regard to Asian populations. These indices may also allow comparisons of future national and international epidemiological studies, as well as the prevention and recognition of obesity, undernutrition, and sarcopenia.

Acknowledgments The authors would like to express their gratitude to all participating individuals. We are grateful to the staff members of the Department of Nuclear Medicine, the First Affiliated Hospital of Jinan University, for excellent technical support.

Author contributions Xiao and Xu were responsible for the conception of this paper. Tang, Shang, and Cheng participated in data collection. Xiao and Guo were responsible for the analysis and interpretation of data. Xiao, Guo, Gong, Tang, Shang, Cheng, and Xu were responsible for the critical review of the article for intellectual content. Guo and Gong were in charge of the statistical expertise. Xu supervised the study. All authors read and approved the final version of the manuscript.

\section{Compliance with ethical standards}

Conflict of interest Z. Xiao, B. Guo, J. Gong, Y. Tang, J. Shang, Y. Cheng, and $\mathrm{H}$. Xu reported no conflict of interest related to the study.

Open Access This article is distributed under the terms of the Creative Commons Attribution 4.0 International License (http://creativecommons.org/licenses/by/4.0/), which permits unrestricted use, distribution, and reproduction in any medium, provided you give appropriate credit to the original author(s) and the source, provide a link to the Creative Commons license, and indicate if changes were made.

\section{References}

1. Thibault R, Genton L, Pichard C (2012) Body composition: why, when and for who? Clin Nutr 31(4):435-447. doi:10.1016/j. clnu.2011.12.011

2. Visser M, Langlois J, Guralnik JM, Cauley JA, Kronmal RA, Robbins J, Williamson JD, Harris TB (1998) High body fatness, but not low fat-free mass, predicts disability in older men and women: the Cardiovascular Health Study. Am J Clin Nutr 68(3):584-590 
3. Keys A, Fidanza F, Karvonen MJ, Kimura N, Taylor HL (2014) Indices of relative weight and obesity. Int J Epidemiol 43(3):655-665. doi:10.1093/ije/dyu058

4. Muller MJ (2013) From BMI to functional body composition. Eur J Clin Nutr 67(11):1119-1121. doi:10.1038/ejen.2013.174

5. VanItallie TB, Yang MU, Heymsfield SB, Funk RC, Boileau RA (1990) Height-normalized indices of the body's fat-free mass and fat mass: potentially useful indicators of nutritional status. Am J Clin Nutr 52(6):953-959

6. Peltz G, Aguirre MT, Sanderson M, Fadden MK (2010) The role of fat mass index in determining obesity. Am J Hum Biol 22(5):639-647. doi:10.1002/ajhb.21056

7. Liu P, Ma F, Lou H, Zhu Y (2014) Utility of obesity indices in screening Chinese postmenopausal women for metabolic syndrome. Menopause 21(5):509-514. doi:10.1097/ GME.0b013e3182a170be

8. Baumgartner RN, Koehler KM, Gallagher D, Romero L, Heymsfield SB, Ross RR, Garry PJ, Lindeman RD (1998) Epidemiology of sarcopenia among the elderly in New Mexico. Am J Epidemiol 147(8):755-763

9. Cruz-Jentoft AJ, Baeyens JP, Bauer JM, Boirie Y, Cederholm T, Landi F, Martin FC, Michel JP, Rolland Y, Schneider SM, Topinkova E, Vandewoude M, Zamboni M (2010) Sarcopenia: European consensus on definition and diagnosis: Report of the European Working Group on Sarcopenia in older people. Age Ageing 39(4):412-423. doi:10.1093/ageing/afq034

10. Toombs RJ, Ducher G, Shepherd JA, De Souza MJ (2012) The impact of recent technological advances on the trueness and precision of DXA to assess body composition. Obes (Silver Spring) 20(1):30-39. doi:10.1038/oby.2011.211

11. Kelly TL, Wilson KE, Heymsfield SB (2009) Dual energy $\mathrm{X}$-ray absorptiometry body composition reference values from NHANES. PLoS ONE 4(9):e7038. doi:10.1371/journal. pone. 0007038

12. Fan B, Shepherd JA, Levine MA, Steinberg D, Wacker W, Barden HS, Ergun D, Wu XP (2014) National Health and Nutrition Examination Survey whole-body dual-energy X-ray absorptiometry reference data for GE Lunar systems. J Clin Densitom 17(3):344-377. doi:10.1016/j.jocd.2013.08.019

13. Hong S, Oh HJ, Choi H, Kim JG, Lim SK, Kim EK, Pyo EY, Oh K, Kim YT, Wilson K, Choi WH (2011) Characteristics of body fat, body fat percentage and other body composition for Koreans from KNHANES IV. J Korean Med Sci 26(12):1599-1605. doi:10.3346/jkms.2011.26.12.1599

14. Guo B, Xu Y, Gong J, Tang Y, Shang J, Xu H (2015) Reference data and percentile curves of body composition measured with dual energy X-ray absorptiometry in healthy Chinese children and adolescents. J Bone Miner Metab 33(5):530-539. doi:10.1007/s00774-014-0615-5

15. Maskarinec G, Erber E, Grandinetti A, Verheus M, Oum R, Hopping BN, Schmidt MM, Uchida A, Juarez DT, Hodges K, Kolonel LN (2009) Diabetes incidence based on linkages with health plans: the multiethnic cohort. Diabetes 58(8):1732-1738. doi: $10.2337 / \mathrm{db} 08-1685$

16. Stommel M, Schoenborn CA (2010) Variations in BMI and prevalence of health risks in diverse racial and ethnic populations. Obes (Silver Spring) 18(9):1821-1826. doi:10.1038/ oby. 2009.472

17. Lee MJ, Wu Y, Fried SK (2013) Adipose tissue heterogeneity: implication of depot differences in adipose tissue for obesity complications. Mol Asp Med 34(1):1-11. doi:10.1016/j. mam.2012.10.001
18. Katzmarzyk PT, Bray GA, Greenway FL, Johnson WD, Newton RL Jr, Ravussin E, Ryan DH, Smith SR, Bouchard C (2010) Racial differences in abdominal depot-specific adiposity in white and African American adults. Am J Clin Nutr 91(1):7-15. doi:10.3945/ajen.2009.28136

19. Cole TJ, Green PJ (1992) Smoothing reference centile curves: the LMS method and penalized likelihood. Stat Med 11(10):1305-1319

20. Gallagher D, Visser M, De Meersman RE, Sepulveda D, Baumgartner RN, Pierson RN, Harris T, Heymsfield SB (1997) Appendicular skeletal muscle mass: effects of age, gender, and ethnicity. J Appl Physiol 83(1):229-239

21. Janssen I, Heymsfield SB, Wang ZM, Ross R (2000) Skeletal muscle mass and distribution in 468 men and women aged 18-88 yr. J Appl Physiol 89(1):81-88

22. Kim MK, Baek KH, Song KH, Il Kang M, Park CY, Lee WY, Oh KW (2011) Vitamin D deficiency is associated with sarcopenia in older Koreans, regardless of obesity: the Fourth Korea National Health and Nutrition Examination Surveys (KNHANES IV) 2009. J Clin Endocrinol Metab 96(10):3250-3256. doi:10.1210/ jc.2011-1602

23. Sanada K, Miyachi M, Tanimoto M, Yamamoto K, Murakami H, Okumura S, Gando Y, Suzuki K, Tabata I, Higuchi M (2010) A cross-sectional study of sarcopenia in Japanese men and women: reference values and association with cardiovascular risk factors. Eur J Appl Physiol 110(1):57-65. doi:10.1007/ s00421-010-1473-z

24. Wells JC (2007) Sexual dimorphism of body composition. Best Pract Res Clin Endocrinol Metab 21(3):415-430. doi:10.1016/j. beem.2007.04.007

25. Pinnick KE, Nicholson G, Manolopoulos KN, McQuaid SE, Valet P, Frayn KN, Denton N, Min JL, Zondervan KT, Fleckner J, McCarthy MI, Holmes CC, Karpe F (2014) Distinct developmental profile of lower-body adipose tissue defines resistance against obesity-associated metabolic complications. Diabetes 63(11):3785-3797. doi:10.2337/db14-0385

26. Reis JP, Loria CM, Lewis CE, Powell-Wiley TM, Wei GS, Carr JJ, Terry JG, Liu K (2013) Association between duration of overall and abdominal obesity beginning in young adulthood and coronary artery calcification in middle age. JAMA 310(3):280-288. doi:10.1001/jama.2013.7833

27. Morimoto Y, Maskarinec G, Conroy SM, Lim U, Shepherd J, Novotny R (2012) Asian ethnicity is associated with a higher trunk/peripheral fat ratio in women and adolescent girls. J Epidemiol 22(2):130-135

28. Kadowaki T, Sekikawa A, Murata K, Maegawa H, Takamiya T, Okamura T, El-Saed A, Miyamatsu N, Edmundowicz D, Kita Y, Sutton-Tyrrell K, Kuller LH, Ueshima H (2006) Japanese men have larger areas of visceral adipose tissue than Caucasian men in the same levels of waist circumference in a populationbased study. Int J Obes (Lond) 30(7):1163-1165. doi:10.1038/ sj.ijo.0803248

29. He Q, Horlick M, Thornton J, Wang J, Pierson RN Jr, Heshka S, Gallagher D (2002) Sex and race differences in fat distribution among Asian, African-American, and Caucasian prepubertal children. J Clin Endocrinol Metab 87(5):2164-2170. doi:10.1210/jcem.87.5.8452

30. Zhou BF (2002) Predictive values of body mass index and waist circumference for risk factors of certain related diseases in Chinese adults-study on optimal cut-off points of body mass index and waist circumference in Chinese adults. Biomed Environ Sci 15(1):83-96 